\title{
Cost per cumulative clinical benefit of biologic therapies for patients with plaque psoriasis: a systematic review
}

\author{
Andrew Blauvelt, MD, MBA; Russel Burge, PhD; William Malatestinic, PharmD, MBA; Alan Brnabic, MSc; \\ Jiaying Guo, MS; Manju Janardhanan, MD; and Baojin Zhu, PhD
}

\section{What is already known about this subject}

- Network meta-analyses comparing cumulative clinical efficacy of biologics in psoriasis reported that ixekizumab and brodalumab have greater cumulative benefits for $\geq 75 \%$ improvement in PASI (PASI 75), $\geq 90 \%$ improvement in PASI (PASI 90), and $100 \%$ improvement in PASI (PASI 100) responses than other biologics with 12-week data.

- Ixekizumab showed greater cumulative benefits than other biologics with 16 -week cumulative efficacy data.

\section{ABSTRACT}

BACKGROUND: Measuring cumulative clinical treatment benefit over time captures speed and magnitude of effects. Assessing the cost of biologics relative to their cumulative clinical benefits versus a single time point represents an alternative to evaluate the value of a given biologic used to treat psoriasis.

OBJECTIVE: To compare cumulative benefit and cost per cumulative benefit of biologics in treatment of moderate to severe psoriasis from a network meta-analysis (NMA).

METHODS: Biologics included in the analysis were ixekizumab, adalimumab, guselkumab,

\section{What this study adds}

- Among biologics, including recently approved biologics such as risankizumab and certolizumab pegol, ixekizumab demonstrated the greatest cumulative clinical benefit for PASI 75 , PASI 90, and PASI 100 responses over a 16-week period.

- Including week 16 doses, ixekizumab demonstrated the lowest cost per cumulative benefit for PASI 100 and lowest discounted cost per cumulative benefit for PASI 75, PASI 90, and PASI 100 responses over a 16-week period.

- Excluding week 16 doses, risankizumab demonstrated the lowest cost per cumulative benefit for PASI 90 and PASI 100, and ixekizumab the lowest discounted cost per cumulative benefit for PASI 90 and PASI 100 responses over a 16-week period.

ustekinumab, secukinumab, risankizumab, and certolizumab pegol. Psoriasis Area and Severity Index (PASI) responses over the initial 16-week treatment period were obtained from 31 articles. Cumulative benefits for PASI 75, PASI 90, and PASI 100 responses were measured as area under the curve (AUC) using the trapezoidal method. Bayesian-based NMA modeled percent maximum AUC through week 16 (\%Max_AUC $\left.{ }_{\mathrm{w}_{16}}\right)$. The AUC estimates over 16 weeks were converted to total skin clearance threshold days achieved for PASI 75, PASI 90, and PASI 100 with each biologic. Cost per cumulative benefit was estimated by multiplying number of doses (per FDA label) by nationally

\section{Author affiliations}

Andrew Blauvelt, MD, MBA, Oregon Medical Research Center, Portland, OR. Russel Burge, PhD, Eli Lilly and Company, Indianapolis, IN, and University of Cincinnati, Cincinnati, $\mathrm{OH}$. William Malatestinic, PharmD, MBA; Alan Brnabic, MSc; Jiaying Guo, MS; and Baojin Zhu, PhD, Eli Lilly and Company, Indianapolis, IN. Manju Janardhanan, MD, Eli Lilly Services India Pvt. Ltd., Bangalore, India.

\section{AUTHOR CORRESPONDENCE:}

Russel Burge, 317.277.9530;

burge_russel_thomas@lilly.com

J Manag Care Spec Pharm. 2021;27(1):84-94

Copyright (C2021, Academy of Managed Care Pharmacy. All rights reserved.

representative discounted wholesale acquisition costs (WACs) for 16 weeks of treatment divided by $\%$ Max_AUC ${ }_{\mathrm{w} 16}$. The primary cost analysis used WACs, including week 16 doses. Co-primary cost analysis used discounted WACs, including week 16 doses. Sensitivity analysis was conducted using WACs and discounted WACs, excluding doses administered at week 16.

RESULTS: Among biologics with available week 16 AUC data for PASI 90 and PASI 100, cumulative benefits over the initial 16-week treatment period ranged from $20.2 \%$ (certolizumab pegol) to $47.0 \%$ (ixekizumab) for PASI 90 and from $7.4 \%$ (adalimumab) to $22.2 \%$ (ixekizumab) for PASI 100. The total 
number of estimated PASI 90 and PASI 100 days achieved over the first 16 weeks of treatment was highest with ixekizumab (53 days and 25 days, respectively). In the primary analysis, guselkumab had the lowest cost per cumulative benefit ( $95 \%$ credible interval [Crl]; \$99,742 [\$89,941-\$111,653]), followed by ixekizumab $(\$ 108,906$ $[\$ 95,928-\$ 126,093])$ and adalimumab $(\$ 111,233[\$ 97,549-\$ 129,022])$ for PASI 90, and ixekizumab had the lowest cost per cumulative benefit $(\$ 230,884[\$ 191,611-\$ 291,115])$, followed by secukinumab $(\$ 238,945$ [\$204,029-\$288,072]) and risankizumab $(\$ 279,968$ $[\$ 250,683-\$ 316,872])$ for PASI 100 responses. In the co-primary analysis, ixekizumab had the lowest discounted cost per AUC $(95 \%$ Crl; $\$ 60,988$ [\$53,719-\$70,612]), followed by guselkumab $(\$ 66,827$ $[\$ 60,260-\$ 74,807])$ and secukinumab $(\$ 69,622[\$ 61,783-\$ 79,786])$ for PASI 90, and ixekizumab had the lowest cost per cumulative benefit $(\$ 129,295$ [\$107,302-\$163,024]), followed by secukinumab $(\$ 148,146$ $[\$ 126,498-\$ 178,605])$ and guselkumab $(\$ 188,190[\$ 166,791-\$ 215,969])$ for PASI 100 responses.

Conclusions: Among biologics studied, ixekizumab demonstrated the greatest cumulative clinical benefit, maintaining the lowest cost per cumulative benefit for PASI 100 responses and lowest discounted cost per cumulative benefit for PASI 90 and PASI 100 responses for moderate to severe psoriasis over the initial 16-week treatment period.

Plaque psoriasis, the most common type of psoriasis, is characterized by thick, red, and scaly plaques that can be either localized to the elbows, knees, scalp or have widespread distribution throughout the body. ${ }^{1}$ In the United States, the prevalence of psoriasis is approximately 3\% (2010 estimates). ${ }^{2}$ Psoriasis can result in cumulative life course impairment due to its substantial effect on all aspects of quality of life. ${ }^{3}$ In addition, numerous comorbidities are associated with psoriasis, including psoriatic arthritis and cardiovascular disease. ${ }^{1}$

The Psoriasis Area and Severity Index (PASI) is often used in clinical trials as an objective measurement of psoriasis severity. With the advent of new biologics that have high potential to achieve clear or almost clear skin, $90 \%$ or more improvement in PASI (i.e., PASI 90) has become the new treatment goal standard in psoriasis. ${ }^{4}$ Indeed, infliximab and newer biologics, such as tildrakizumab, ixekizumab, secukinumab, risankizumab, brodalumab, and guselkumab, are associated with achievement of PASI 90 responses in $>50 \%$ of patients. ${ }^{5-11}$ Furthermore, when surveyed, patients highlight "total skin clearance" or PASI 100 as the most important goal of treatment. Achieving total skin clearance makes patients happier, more confident, and feel better compared with less than complete skin clearance. ${ }^{12}$ Patients also want treatments to work fast, expecting to achieve $50 \%$ clear skin in 2 weeks and $100 \%$ clear skin by 4 weeks. ${ }^{13,14}$

Endpoints such as PASI 90 and PASI 100 are measured at specific time points in clinical trials and reflect the treatment response at that point of time. Unlike PASI responses, measuring "cumulative clinical benefit" accounts for time to onset, magnitude of treatment effects, and durability of responses; this is assessed by plotting patient-level area under the curve (AUC) efficacy data over a defined time period..$^{15}$

The total cost burden of psoriasis in the United States has been estimated to be $\$ 112$ billion to $\$ 135$ billion per year, where direct health care costs (e.g., medical evaluations, hospitalizations, prescription medications, and phototherapy) comprised $\$ 51.7$ billion to $\$ 63.2$ billion of the overall total. ${ }^{16}$ Biologic therapies, particularly newer ones, offer great potential efficacy but at relatively high costs in terms of wholesale acquisition costs (WACs) per dose. WACs are the manufacturer list prices to wholesalers, but WAC prices do not include rebates or discounts given to health care payers so do not accurately correspond to final transaction prices paid. ${ }^{17}$ Of note, most cost-effectiveness or other cost analyses-such as cost per PASI response, cost per number needed to treat, and cost per additional PASI responder-have relied on WAC prices. ${ }^{18-20}$

The Institute for Clinical and Economic Review (ICER) developed a final evidence report (dated August 3, 2018) on effectiveness and value of targeted immunomodulators in psoriasis. ${ }^{21}$ This report was based on data from randomized controlled trials and high-quality systematic reviews, included sensitivity analysis, and used a health sector perspective with longer time horizons (10-year and lifetime). Ixekizumab (7.42 quality-adjusted life-years [QALYs]) was the most effective treatment, whereas apremilast was least effective (6.79 QALYs). The most expensive treatment was guselkumab $(\$ 342,000)$ and the least expensive was apremilast $(\$ 215,000)$. Cost per QALY was highest for certolizumab pegol $(\$ 188,000)$ and lowest for brodalumab $(\$ 131,000)$. Ixekizumab ranked fourth in the list at $\$ 142,000$ per QALY. Brodalumab was the most cost-effective option among interleukin (IL)-17 inhibitors. Initiation with IL-17 inhibitors or infliximab was cost-effective at higher willingness-to-pay thresholds, while apremilast was cost-effective at moderate thresholds..$^{21}$

Here, we measure cumulative benefits in PASI responses over the initial 16-week psoriasis treatment period for 7 biologics, using a network meta-analysis (NMA). ${ }^{22}$ We then compare the relative cost impact of the biologics by measuring cost per cumulative benefit for each treatment, using WACs, and after applying nationally representative discount rates. 


\begin{tabular}{|c|c|c|c|c|c|c|}
\hline \multirow[b]{3}{*}{ Study/Treatment } & \multicolumn{6}{|c|}{ Response Rate Observed in Study, \% } \\
\hline & \multicolumn{2}{|c|}{ PASI 75} & \multicolumn{2}{|c|}{ PASI 90} & \multicolumn{2}{|c|}{ PASI 100} \\
\hline & Week 12 & Week 16 & Week 12 & Week 16 & Week 12 & Week 16 \\
\hline \multicolumn{7}{|l|}{ CIMPACT $^{23}$} \\
\hline Etanercept 50 mg biweekly & 53.3 & - & 27.1 & - & - & - \\
\hline Certolizumab pegol $200 \mathrm{mg}$ Q2W & 61.3 & 68.2 & 31.2 & 39.8 & - & - \\
\hline Certolizumab pegol $400 \mathrm{mg}$ Q2W & 66.7 & 74.7 & 34.0 & 49.1 & - & - \\
\hline Placebo & 5.0 & 3.8 & 0.2 & 0.3 & - & - \\
\hline \multicolumn{7}{|l|}{ CIMPASI- $1^{24}$} \\
\hline Certolizumab pegol $200 \mathrm{mg}$ Q2W & 57.1 & 66.5 & 25.9 & 35.8 & - & - \\
\hline Certolizumab pegol $400 \mathrm{mg}$ Q2W & 64.2 & 75.8 & 30.0 & 43.6 & - & - \\
\hline Placebo & 2.5 & 6.5 & 0.3 & 0.4 & - & - \\
\hline \multicolumn{7}{|l|}{ CIMPASI-2 $2^{24}$} \\
\hline Certolizumab pegol $200 \mathrm{mg}$ Q2W & 77.4 & 81.4 & 44.0 & 52.6 & - & - \\
\hline Certolizumab pegol $400 \mathrm{mg}$ Q2W & 76.3 & 82.6 & 52.0 & 55.4 & - & - \\
\hline Placebo & 13.5 & 11.6 & 6.3 & 4.5 & - & - \\
\hline \multicolumn{7}{|l|}{ ultiMMa-125 } \\
\hline Risankizumab $150 \mathrm{mg}$ Q12W & - & - & 68.0 & 75.0 & 27.3 & 35.9 \\
\hline Ustekinumab 45/90 mg & - & - & 45.0 & 42.0 & 13.9 & 12.0 \\
\hline Placebo & - & - & 3.0 & 5.0 & 0 & 0 \\
\hline \multicolumn{7}{|l|}{ ultiMMa-2 ${ }^{25}$} \\
\hline Risankizumab $150 \mathrm{mg}$ Q12W & - & - & 62.0 & 75.0 & 37.9 & 50.7 \\
\hline Ustekinumab $45 / 90 \mathrm{mg}$ & - & - & 47.0 & 47.0 & 23.2 & 24.2 \\
\hline Placebo & - & - & 3.0 & 2.0 & 2.0 & 2.0 \\
\hline
\end{tabular}

Note: Hyphen indicates no data available.

NMA = network meta-analysis; PASI = Psoriasis Area and Severity Index; PASI 75= $=75 \%$ improvement in PASI; PASI $90=\geq 90 \%$ improvement in PASI; PASI 100 $=100 \%$ improvement in PASI; Q2W=every 2 weeks; $\mathrm{Q} 12 \mathrm{~W}=$ every 12 weeks

\section{MEASUREMENT OF CUMULATIVE CLINICAL BENEFITS}

Missing values for PASI 75, PASI 90, and PASI 100 response rates were imputed using nonresponder imputation in all the trials, except in 3 trials of certolizumab pegol where multiple imputation was used. Cumulative benefits in PASI 75, PASI 90, and PASI 100 response rates were measured as AUC over the initial 16-week treatment period $\left(\mathrm{AUC}_{\mathrm{W} 16}\right)$ using the trapezoidal rule. The proportion of maximum cumulative benefit (Max_AUC $\left.{ }_{\mathrm{W} 16}\right)$ in PASI 75, PASI 90, and PASI 100 for each therapy was estimated by dividing the $\mathrm{AUC}_{\mathrm{W} 16}$ by the maximum AUC, where maximum AUC equals the number of weeks of treatment. This was then converted to \%Max_AUC ${ }_{\mathrm{W} 16}$ by multiplying by 100 . The number of PASI 75 , PASI 90, and PASI 100 skin clearance threshold days achieved with each biologic was also estimated by multiplying \%Max_AUC ${ }_{\mathrm{W} 16}$ for the respective PASI response groups of each biologic by 112 (16 weeks=112 days).

\section{COMPARISON OF CUMULATIVE CLINICAL BENEFITS}

In our analysis, a Bayesian NMA was conducted to model \%Max_AUC ${ }_{\mathrm{W} 16}$ along with \%MAX_AUC ${ }_{\mathrm{W} 12} \cdot{ }^{22,26}$ An NMA compares multiple interventions simultaneously by analyzing studies and making different comparisons in the same analysis. ${ }^{27}$ An NMA combines direct and indirect evidence to strengthen inferences concerning relative effects of treatments. ${ }^{28}$ This allows for multiple treatment comparisons to be made where there may not exist direct evidence and strengthens the comparisons where direct evidence is available. Where various treatment options are available, NMAs are increasingly being used to inform decision making. This analysis is an update to the analysis done by Warren et al. and includes certolizumab pegol and risankizumab, 


\section{FIGURE 1 NMA Diagram for PASI 90 Responses at Week 16}

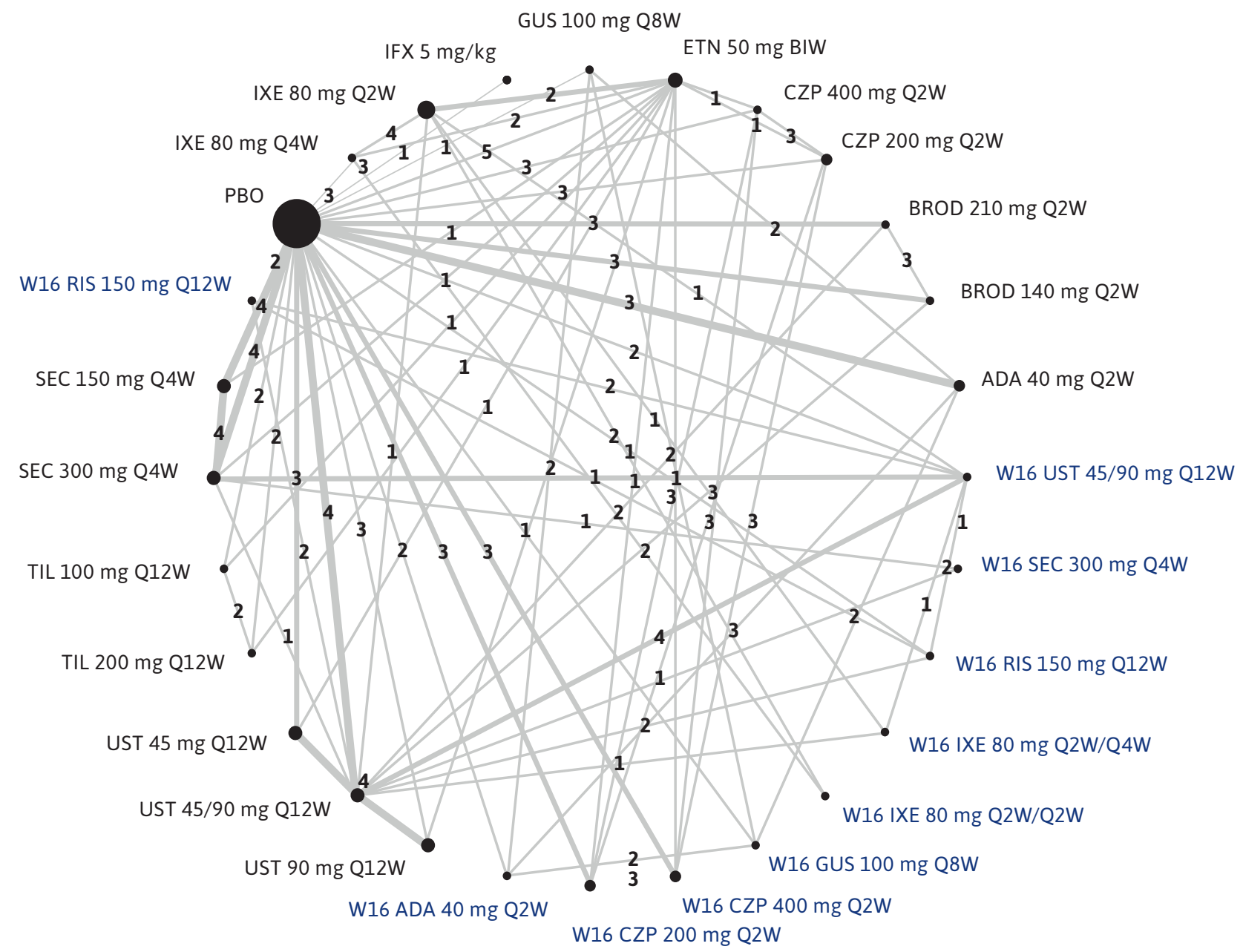

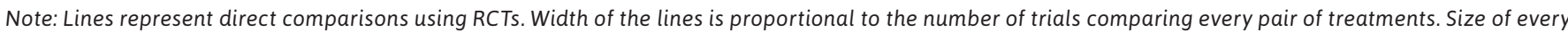
circle is proportional to the number of randomly assigned participants (i.e., sample size). Numbers represent the number of RCTs included in each comparison. Blue highlighted text indicates biologics included in the current analyses.

$A D A=$ adalimumab; $B I W=$ biweekly; $B R O D=$ brodalumab; $C Z P=$ certolizumab pegol; $E T N=$ etanercept; IFX =infliximab; IXE =ixekizumab; GUS = guselkumab; $N M A=$ network meta-analysis; PASI = Psoriasis Area and Severity Index; PBO=placebo; Q2W=every 2 weeks; Q4W=every 4 weeks; Q8W=every 8 weeks; $\mathrm{Q} 12 \mathrm{~W}=$ every 12 weeks; $R C T s=$ randomized controlled trials; $R I S=$ risankizumab; $S E C=$ secukinumab; TIL = tildrakizumab; UST=ustekinumab.

the newer IL-23 biologic treatments for psoriasis. ${ }^{22}$ Fixed and random effects models were used to analyze the data. The NMA was performed using Just Another Gibbs Sampler (JAGS) version 4.2.0 software via R2jags in R3.6.2. ${ }^{29,30}$ For an illustration of how the trapezium rule is used to generate the AUC estimates from the data generated in the NMA, see Supplementary Figure 1 (available in online article).

Parameters used for the modeling included a burn-in and post burn-in sample of 350,000 iterations for each, a thinning value of 3 , three chains, a uniform $(0,5)$ prior distribution for between-trial treatment effect SD, and a uniform $(0,2)$ prior distribution for baseline between-trial SD. Normal independent Bayesian models were assessed for fit, using the deviance information criterion (DIC), as well as comparing the number of data points to the residual deviance. DIC is a useful approach for comparing between fixed and random effects models for similar data. ${ }^{27,31}$ 


\section{TABLE 2} Percentage of Maximum AUCa and Skin Clearance
Threshold Days with Each Biologic During 16-Week Period

\begin{tabular}{|c|c|c|c|}
\hline & Medi & $\begin{array}{l}\text { \%Max_AUC }{ }_{w_{16}} \\
\text { (95\% Credible In }\end{array}$ & rvals) \\
\hline & PASI 75 & PASI 90 & PASI 100 \\
\hline Ixekizumab 80 mg Q2W/Q4W & $66.2(60.2-72.4)$ & $47.0(40.6-53.4)$ & $22.2(17.6-26.7)$ \\
\hline Adalimumab 40 mg Q2W & $44.3(41.4-47.1)$ & $25.0(21.5-28.5)$ & $7.4 \quad(6.1-8.8)$ \\
\hline Guselkumab 100 mg Q8W & $52.3(48.8-55.9)$ & $34.3(30.6-38.0)$ & $12.2(10.6-13.7)$ \\
\hline Ustekinumab 45/90 mg Q12W & $42.6(38.5-46.8)$ & $23.2(19.9-26.4)$ & $8.9 \quad(7.3-10.6)$ \\
\hline Secukinumab 300 mg Q4W & $57.1 \quad(52.4-61.9)$ & $39.5(34.4-44.5)$ & $18.6(15.4-21.7)$ \\
\hline Risankizumab $150 \mathrm{mg}$ Q12W & - & $37.7(34.2-41.3)$ & $17.0(15.0-19.0)$ \\
\hline Certolizumab pegol 400 mg Q2W & $40.9(36.9-45.0)$ & $20.2(16.7-23.8)$ & - \\
\hline
\end{tabular}

Skin clearance threshold days

\begin{tabular}{l|rc|rc|rc}
\hline Ixekizumab $80 \mathrm{mg}$ Q2W/Q4W & 74.2 & $(67.4-81.0)$ & 52.7 & $(45.5-59.8)$ & 24.8 & $(19.7-29.9)$ \\
\hline Adalimumab $40 \mathrm{mg}$ Q2W & 49.6 & $(46.4-52.8)$ & 28.0 & $(24.1-31.9)$ & 8.3 & $(6.8-9.8)$ \\
\hline Guselkumab $100 \mathrm{mg}$ Q8W & 58.6 & $(54.7-62.6)$ & 38.4 & $(34.3-42.6)$ & 13.7 & $(11.9-15.4)$ \\
\hline Ustekinumab 45/90 mg Q12W & 47.7 & $(43.1-52.4)$ & 26.0 & $(22.3-29.6)$ & 10.0 & $(8.1-11.9)$ \\
\hline Secukinumab $300 \mathrm{mg}$ Q4W & 64.0 & $(58.7-69.3)$ & 44.2 & $(38.6-49.8)$ & 20.8 & $(17.2-24.3)$ \\
\hline Risankizumab 150 mg Q12W & - & 42.2 & $(38.3-46.2)$ & 19.0 & $(16.8-21.2)$ \\
\hline Certolizumab pegol 400 mg Q2W & 45.8 & $(41.3-50.4)$ & 22.6 & $(18.7-26.7)$ & \multicolumn{2}{c}{-} \\
\hline
\end{tabular}

aPASI 100 results from fixed effects models; PASI 90 and PASI 75 results from random effects models. $A U C=$ are $a$ under the curve; $P A S I=P$ soriasis Area and Severity Index; PASI $75=\geq 75 \%$ improvement in PASI score; PASI $90=\geq 90 \%$ improvement in PASI score; PASI 100=100\% improvement in PASI score; Q2W=every 2 weeks; $\mathrm{Q} 4 \mathrm{~W}=$ every 4 weeks; $\mathrm{Q} 8 \mathrm{~W}=$ every 8 weeks; $\mathrm{Q} 12 \mathrm{~W}=$ every 12 weeks.

Convergence for all models was assessed using trace plots as modified by Brooks and Gelman (1998). ${ }^{32}$ We report the percentage of maximum AUC with each therapy at week 16 based on the random-effects models for PASI 75 and PASI 90 and the fixed effects model for PASI 100. If the random effects models did not converge or if the model fit was better for the fixed effects, then the results from fixed-effects models were used. Models with lower DIC and with the number of data points similar to the residual deviance, in combination with good diagnostic and convergent properties, were reported. ${ }^{26}$

\section{CALCULATION OF COST PER CUMULATIVE BENEFIT}

Dosing of each treatment was obtained from U.S. prescribing information. ${ }^{33-39}$ For each biologic, unit dose cost was based on WAC, with and without incorporating drug discount costs. Discount rates were obtained from the ICER final evidence report dated August 3, 2018; WAC data were obtained from RED BOOK. ${ }^{21,40}$ Discount rates were not available for risankizumab. Total costs over the initial 16-week treatment period were calculated by multiplying the number of doses over this time and WAC (or nationally representative discounted WACs) per dose in U.S. dollars for each treatment. Cost per cumulative benefit was obtained by dividing the cost over 16 weeks by \%Max_AUC ${ }_{\mathrm{W} 16}$ for each treatment.

Efficacy estimates from the randomized controlled trials were based on the last day of exposure from the last injection in the induction period. Thus, the 16-week efficacy measurement for guselkumab would include the week 8 injection but not the subsequent week 16 injection. Similarly, for ixekizumab (induction period of 12 weeks), the week 16 efficacy measurement was estimated at the end of the 4-week exposure from the week 12 injection and did not include the week 16 injection. Primary cost analysis was conducted using WACs, including the doses administered at week 16 . Co-primary analysis was conducted using discounted WACs, including the doses administered at week 16 . Sensitivity analyses were performed using WACs and discounted WACs, excluding the week 16 doses.

\section{Results}

A total of 31 articles were included in the NMA. Figure 1 provides an example of an NMA diagram using PASI 90 data at week 16 . The biologics included in the current analysis were ixekizumab, adalimumab, guselkumab, ustekinumab, secukinumab, risankizumab, and certolizumab pegol. WAC per dose (as of June 1, 2020) were as follows: ixekizumab: $\$ 5,690$ (9 of the $80 \mathrm{mg}$ dose/16 weeks); adalimumab: $\$ 2,778$ (10 of the $40 \mathrm{mg}$ dose/16 weeks); guselkumab: \$11,392 (3 of the $100 \mathrm{mg}$ dose/16 weeks); ustekinumab: \$14,554 (3 doses by week 16 assuming 27.1\% with weight $>100 \mathrm{~kg})^{41}$; secukinumab: $\$ 5,541$ (16 of the $150 \mathrm{mg}$ dose/16 weeks); risankizumab: $\$ 7,921$ (6 of the $75 \mathrm{mg}$ dose by week 16); and certolizumab pegol: $\$ 4,630$ (9 of the $400 \mathrm{mg}$ dose/16 weeks). On excluding week 16 dose, the number of doses reduced by 1 dose for ixekizumab, ustekinumab, 


\section{FIGURE 2 Cost per AUC for PASI 75, PASI 90, and PASI 100 Responses (Including Week 16 Doses)}

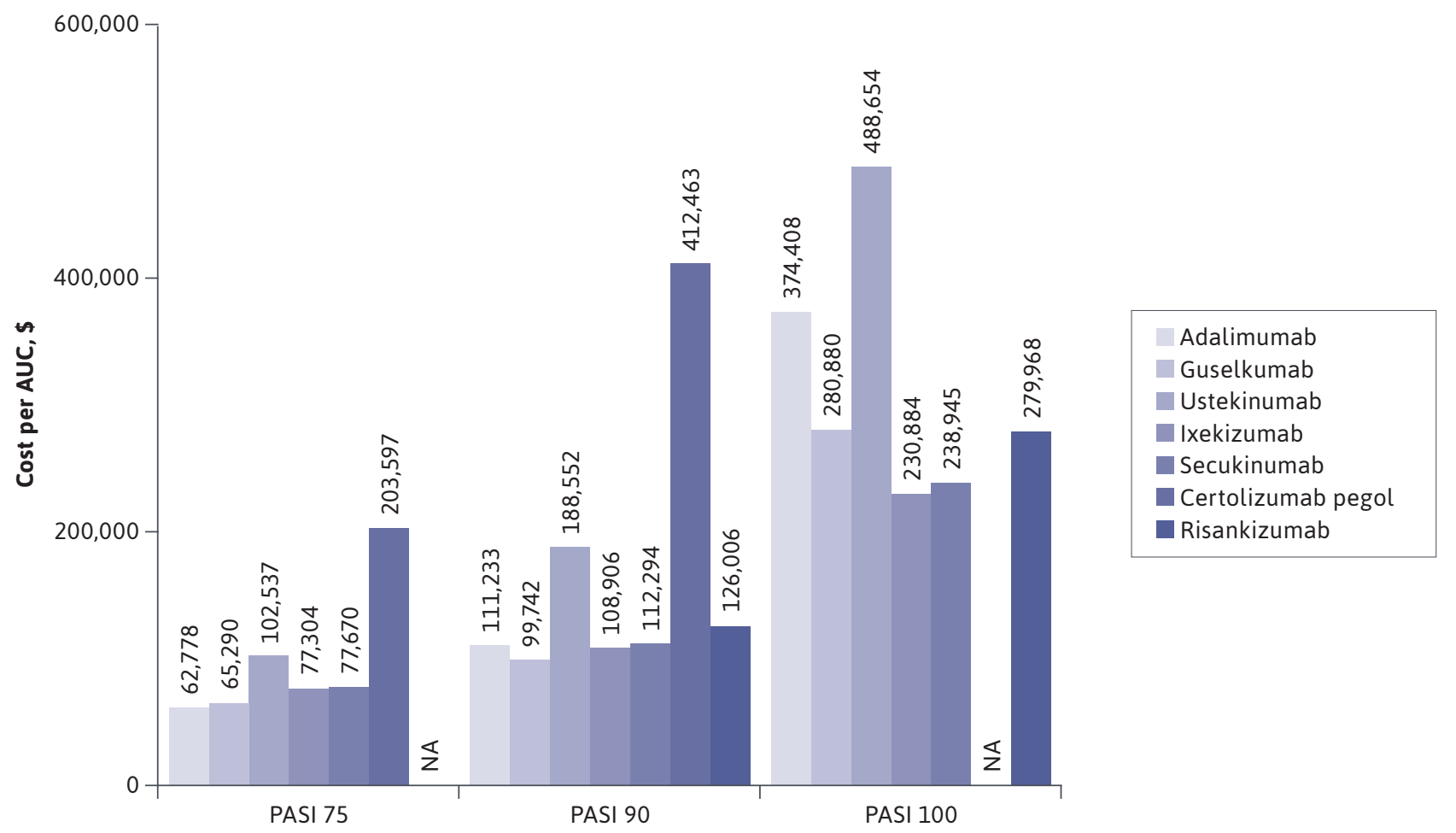

$A U C=$ area under the curve; $N A=$ data not available; $P A S I=$ ssoriasis Area and Severity Index; $P A S I 75=\geq 75 \%$ improvement in PASI; PASI $90=\geq 90 \%$ improvement in PASI; PASI $100=100 \%$ improvement in PASI.

and certolizumab pegol each and 2 doses for secukinumab and risankizumab each. Published discount rates were ixekizumab: $44 \%$, adalimumab: $31 \%$, guselkumab: $33 \%$, ustekinumab: $27 \%$, secukinumab: $38 \%$, and certolizumab pegol: $36 \%{ }^{21}$ After applying discount rates, WAC per dose were as follows: ixekizumab: \$3,186, adalimumab: \$1,917, guselkumab: \$7,632, ustekinumab: \$10,624, secukinumab: \$3,436, and certolizumab pegol: \$2,963.

\section{CUMULATIVE BENEFIT OF BIOLOGICS}

Among the biologics studied, ixekizumab showed the greatest cumulative benefits over 16 weeks for PASI 90 and PASI 100 responses; the \%Max_AUC ${ }_{\mathrm{W} 16}$ were $47.0 \%$ for PASI 90 and 22.2\% for PASI 100 (Table 2). Secukinumab ranked second with \%Max_AUC ${ }_{\mathrm{W} 16}$ values of $39.5 \%$ for PASI 90 and $18.6 \%$ for PASI 100 responses. The cumulative benefit was lowest for certolizumab pegol for PASI 90 and adalimumab for PASI 100 responses. For PASI 75 responses, the cumulative benefit was greatest for ixekizumab and lowest for certolizumab pegol; the \%Max_AUC ${ }_{\mathrm{W} 16}$ was $66.2 \%$ and $40.9 \%$, respectively (Table 2 ).

The total number of PASI 90 days achieved over the initial 16-week treatment period ranged from 23 days for certolizumab pegol to 53 days for ixekizumab (Table 2). The total number of PASI 100 days ranged from 8 days with adalimumab to 25 days with ixekizumab. Ixekizumab was also associated with a greater number of PASI 75 days achieved over the 16-week period (Table 2).

\section{PRIMARY ANALYSIS: WHOLESALE ACQUISITION COSTS INCLUDING WEEK 16 DOSES}

Ixekizumab had the lowest cost (95\% credible interval [CrI]) per cumulative benefit of $\$ 230,884$ (\$191,611-\$291,115) for PASI 100 responses (Figure 2). For PASI 75, adalimumab had the lowest cost per cumulative benefit (\$62,778 [\$58,962$\$ 67,050])$, and for PASI 90, guselkumab had the lowest cost per cumulative benefit (\$99,742 [\$89,941-\$111,653]; Figure 2). Certolizumab pegol had the highest cost per cumulative 


\section{FIGURE 3 Cost per AUC After Incorporating Discount Rates for PASI 75, PASI 90, and PASI 100 Responses (Including Week 16 Doses)}

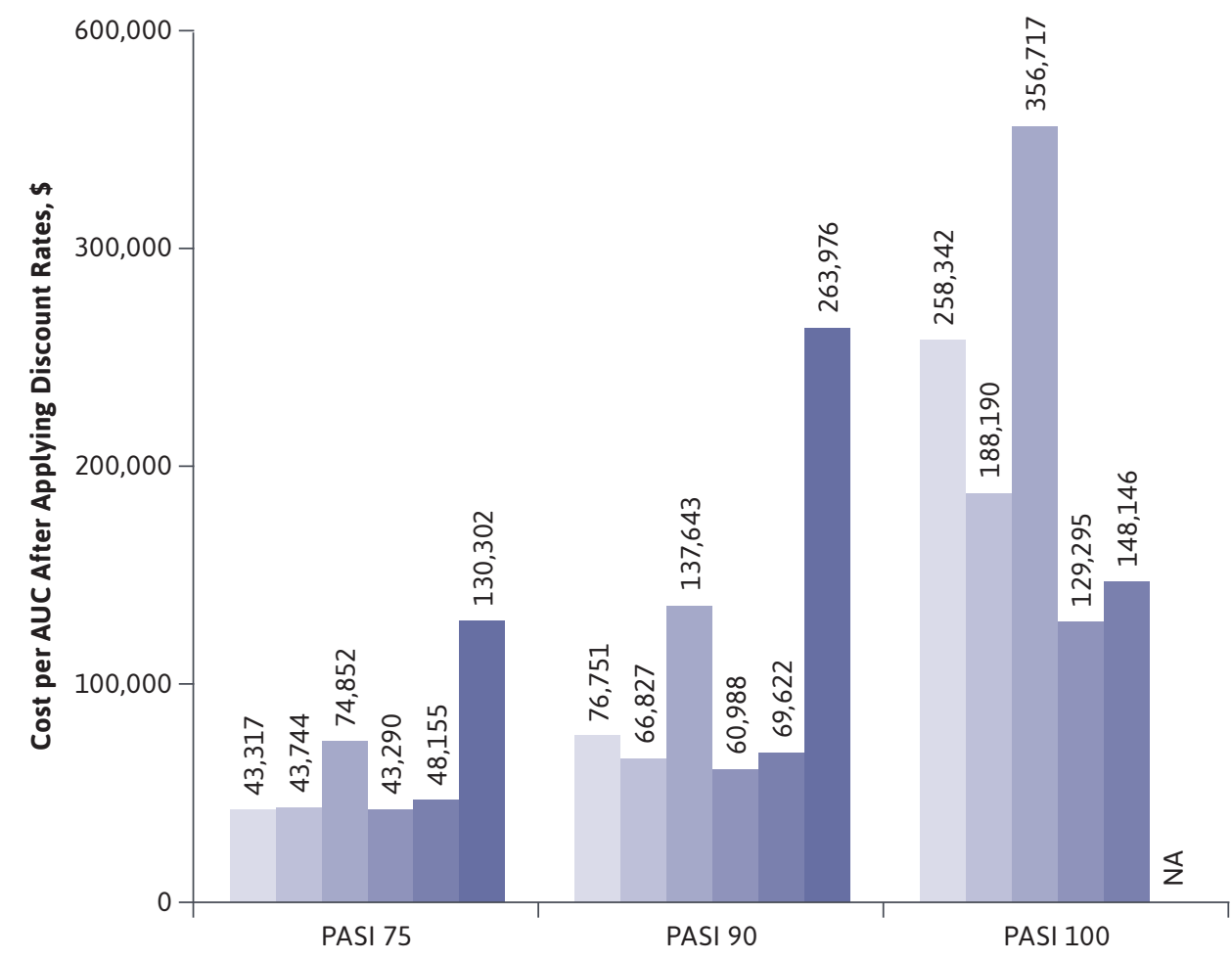

Adalimumab

Guselkumab

Ustekinumab

Ixekizumab

Secukinumab

Sertolizumab pegol

AUC = area under the curve; NA = data not available; PASI = Psoriasis Area and Severity Index; PASI $75=\geq 75 \%$ improvement in PASI; PASI $90=\geq 90 \%$ improvement in PASI; PASI $100=100 \%$ improvement in PASI.

benefit (\$412,463 [\$349,856-\$499,199]) for PASI 90 response, and ustekinumab had the highest cost per cumulative benefit $(\$ 488,654$ [\$411,859-\$601,642]) for PASI 100 response (Figure 2).

\section{CO-PRIMARY ANALYSIS: DISCOUNTED WHOLESALE ACQUISITION COSTS INCLUDING WEEK 16 DOSES}

Ixekizumab had the lowest cost (95\% CrI) per cumulative benefit of $\$ 43,290(\$ 39,629-\$ 47,671)$ for PASI 75 , $\$ 60,988$ $(\$ 53,719-\$ 70,612)$ for PASI 90 , and $\$ 129,295$ (\$107,302$\$ 163,024)$ for PASI 100 responses (Figure 3). Certolizumab pegol had the highest cost per cumulative benefit of \$263,976 $(\$ 223,908-\$ 319,487)$ for PASI 90 response, and ustekinumab had the highest cost per cumulative benefit of $\$ 356,717$ $(\$ 300,657-\$ 439,199)$ for PASI 100 response (Figure 3). The range of discounted costs $(-10 \%$ and $+10 \%)$ is presented in Supplementary Table 1 (available in online article).

\section{SENSITIVITY ANALYSIS: WHOLESALE ACQUISITION COSTS EXCLUDING WEEK 16 DOSES}

Risankizumab had the lowest cost (95\% CrI) per cumulative benefit of $\$ 84,004$ ( $\$ 76,746-\$ 92,754$ ) for PASI 90 and $\$ 186,645$ (\$167,122-\$211,248) for PASI 100 responses (Supplementary Table 2, available in online article). Adalimumab had the lowest cost per cumulative benefit of $\$ 62,778$ (\$58,962-\$67,050) for PASI 75 responses. Certolizumab pegol had the highest cost per cumulative benefit of $\$ 366,633$ (\$310,984-\$443,733) for PASI 90 response, and adalimumab had the highest cost per cumulative benefit of $\$ 374,408$ (\$316,348-\$459,026) for PASI 100 response (Supplementary Table 2).

\section{SENSITIVITY ANALYSIS: DISCOUNTED WHOLESALE ACQUISITION COSTS EXCLUDING WEEK 16 DOSES}

Ixekizumab had the lowest cost $(95 \% \mathrm{CrI})$ per cumulative benefit of $\$ 38,480(\$ 35,225-\$ 42,374)$ for PASI $75, \$ 54,211$ $(\$ 47,751-\$ 62,766)$ for PASI 90, and $\$ 114,929$ (\$95,380$\$ 144,910)$ for PASI 100 responses (Supplementary Table 2). 
Certolizumab pegol had the highest cost per cumulative benefit of $\$ 234,645$ (\$199,029-\$283,989) for PASI 90, and adalimumab had the highest cost per cumulative benefit of $\$ 258,342$ (\$218,280-\$316,728) for PASI 100 response (Supplementary Table 2).

\section{Discussion}

We used the AUC to assess cumulative benefits for PASI 75, PASI 90, and PASI 100 responses for psoriasis biologics over the initial 16-week treatment. The percent maximum AUC was then determined and compared between biologics using an NMA. The AUC estimates over 16 weeks were converted to total healthy-state days achieved for PASI 75, PASI 90, and PASI 100 for each biologic. Among the biologics studied, ixekizumab showed the greatest cumulative benefit over the initial 16-week treatment period for PASI 75, PASI 90, and PASI 100 responses. The total number of PASI 75, PASI 90, and PASI 100 days achieved over the 16-week period was also greater with ixekizumab compared with the other biologics.

In the NMA by Warren et al., ${ }^{22}$ greater cumulative benefits for PASI 75, PASI 90, and PASI 100 responses were demonstrated with ixekizumab at 16 weeks than other biologics; however, risankizumab and certolizumab pegol were not included in the analysis. Biologics with 12-week data, such as tildrakizumab, brodalumab, etanercept, and infliximab, were also part of this analysis. Greater cumulative benefits were observed at 12 weeks with ixekizumab and brodalumab compared with other biologics with 12 -week data. ${ }^{22}$

Demonstration of cumulative benefits of medications using patient-level AUC response data was introduced by Armstrong et al. (2017). ${ }^{15}$ Blauvelt et al. (2020) used data from a head-to-head trial of ixekizumab and ustekinumab in patients with moderate to severe plaque psoriasis to compare the cumulative benefits of each drug over a 52-week period. ${ }^{42}$ Ixekizumab was associated with consistent and significantly greater cumulative benefits in PASI 90 (68.9\% vs. $46.5 \%)$ and PASI 100 (41.1\% vs. $23.4 \%)$ response rates, as well as in Itch Numeric Rating Scale and Dermatology Life Quality Index (DLQI) scores. ${ }^{42}$ An NMA was used in the current analysis, however, because of the lack of head-to-head trials for comparison.

The higher efficacy of ixekizumab in achieving clear/ near clear skin has been demonstrated in other NMAs as well. ${ }^{43,44}$ Jabbar-Lopez et al. (2017) estimated cumulative ranking probabilities and used surface under the cumulative ranking curve (SUCRA) to rank treatments. ${ }^{43}$ Using the measure of clear/nearly clear skin, ixekizumab ranked the highest among other biologics, whereas using the measure of DLQI improvement, secukinumab was the best among biologics. ${ }^{43}$ Similarly, in another NMA using SUCRA values to rank treatments, briakinumab, infliximab, ixekizumab, and brodalumab were identified as having high efficacy among the 13 biologics studied. ${ }^{44}$

Psoriasis is a chronic condition that requires long-term treatment. It is clear that treatment goals differ between clinicians and patients. ${ }^{15}$ From the patient's perspective, the ultimate goals are clear skin and rapid relief from symptoms with treatment. ${ }^{45}$ Although biologics are highly effective in the management of psoriasis, the cost of these treatments is high relative to nonbiologic treatments, and increased use of biologics may add to the total societal cost burden of psoriasis.

To better understand the potential value of biologic treatments, we used the concept of cost per cumulative benefit for each biologic over the initial 16-week treatment period. The WAC per dose in U.S. dollars was used for this purpose, and cost analyses were performed, with and without incorporating discount rates, for each PASI response. Primary and sensitivity cost analyses were conducted including and excluding week 16 doses, respectively. In the primary analysis (including week 16 doses), cost per cumulative benefit was lowest for ixekizumab for PASI 100 responses. In the co-primary analysis, the discounted cost per cumulative benefit was lowest for ixekizumab for PASI 75, PASI 90, and PASI 100. In the sensitivity analysis (excluding week 16 doses), cost per cumulative benefit was lowest for risankizumab for both PASI 90 and PASI 100 responses. The discounted cost per cumulative benefit was lowest for ixekizumab for PASI 75, PASI 90, and PASI 100.

Several studies report cost-effectiveness among biologics. ${ }^{18-21,46,47}$ Ravasio et al. (2019) demonstrated ixekizumab to have the lowest cost per responder for PASI 90 and PASI 100 responses among the 5 biologics studied (etanercept, adalimumab, secukinumab, ixekizumab, and ustekinumab). ${ }^{20}$ The differences in cost per responder between ixekizumab and other biologics were greater at higher PASI response levels. Using efficacy data from the UNCOVER trials, it was estimated that ixekizumab once every 2 weeks had lower cost per additional responder for PASI 90 (\$46,299 vs. \$120,720) and PASI 100 (\$80,710 vs. \$404,695) compared with etanercept biweekly. ${ }^{46}$ The number needed to treat for ixekizumab for achieving an additional responder for PASI 90 and PASI 100 responses relative to placebo were lower than other biologics (adalimumab, etanercept, ustekinumab, and secukinumab). ${ }^{19}$

The strengths of our study include the use of Bayesian NMA techniques to estimate comparative efficacy across several biologic therapies, including the most recent anti-IL-17 and anti-IL-23 classes, at multiple time points. Furthermore, the NMA results were used to generate 
comparative estimates of cumulative benefits for each therapy, which were expressed as percentage of maximum AUC over 16 weeks, as well as the number of PASI 75, PASI 90, and PASI 100 days achieved during this period.

The analysis focused on week 16 AUC and associated costs because week 16 was the primary endpoint for many of the phase 3 trials for the latest biologics approved for psoriasis. Response trajectories differ among biologics, thus, the AUC at week 16 may provide a more balanced comparison on different biologic cumulative benefits and associated costs. The cost per AUC was calculated incorporating nationally representative payer discount rates to more closely reflect the actual costs incurred by payers.

\section{LIMITATIONS}

This study has some limitations to consider. Infliximab, brodalumab, tildrakizumab and etanercept were not included in this analysis, since week 16 data were not available for these biologics. In addition, risankizumab was not included in analyses that incorporated discounted WACs because of the absence of published data on the discount rate for this recently approved biologic. Discount rates used for cost analyses were based on the ICER report dated August 2018. ${ }^{21}$ These published rates were used because they were available at the time of the study. Since discount rates change over time, results need to be interpreted with caution. Current WACs have been used in our study.

While the studies included in these analyses had similar inclusion/exclusion criteria, a limitation of this study is that the NMA models did not adjust for baseline risk; however, inspection of all placebo response rates indicate relatively consistent effects across all studies and outcomes. For all phase 3 studies included in this NMA, only a small proportion of the data were missing $(<10 \%)$ by week 16 . The data from each of the studies included in the NMA were all imputed using nonresponder imputation. The results would be expected to be consistent if other data imputation methods were applied, provided that the imputation method applied was the same for every study.

Another limitation is that the trapezium rule was used, which assumes a linear increase in probability of achieving PASI 75, PASI 90, and PASI 100 from week 0 to week 16 . Each study required data at weeks $4,8,12$, and 16 to be included in the analysis. Instability with some NMA models was observed due to zero counts in the placebo arms for PASI 90 and PASI 100 for some studies; however, this issue was overcome by treating the outcome as normally distributed and fitting the baseline and treatment models separately.

This study does not assess the meaningful effect on quality of life, safety of treatments, or cumulative benefits over a time period longer than 16 weeks of treatment, since these were beyond the scope of this project. Future research is warranted to assess these parameters.

Since the data for the NMA analysis have been taken from clinical trials, they may limit the generalizability of results and may not be representative of the general population. In a large real-world cohort, patients who may be ineligible for psoriasis trials differed in important respects (e.g., comorbidities, previous treatments) from their trial-eligible counterparts. ${ }^{48}$ In the absence of head-to-head trials, however, NMAs can serve as a guide for clinicians in making treatmentrelated decisions. Newer model-based approaches could be used to investigate generalizability of the results in future studies. ${ }^{49}$

\section{Conclusions}

Ixekizumab showed the greatest cumulative benefits in terms of potential PASI 75, PASI 90, and PASI 100 threshold days achieved among the biologics studied. After incorporating discount rates, ixekizumab demonstrated lower costs per cumulative benefits across PASI thresholds versus other biologics in this analysis. These results may be useful for clinicians and payers when deciding on cost-effective treatment options for patients with moderate to severe plaque psoriasis.

\section{DISCLOSURES}

This study was funded by Eli Lilly and Company (Indianapolis, IN). Blauvelt has served as a scientific adviser and/or clinical study investigator for AbbVie, Aclaris, Almirall, Arena, Athenex, Boehringer Ingelheim, Bristol-Myers Squibb, Dermavant, Dermira, Eli Lilly and Company, Forte, Galderma, Incyte, Janssen, Leo, Novartis, Ortho, Pfizer, Rapt, Regeneron, Sandoz, Sanofi Genzyme, Sun Pharma, and UCB Pharma and as a paid speaker for AbbVie. Burge, Zhu, Malatestinic, Brnabic, Guo, and Janardhanan are employees and shareholder of Eli Lilly and Company.

\section{REFERENCES}

1. Feldman SR, Goffe B, Rice G, et al. The challenge of managing psoriasis: unmet medical needs and stakeholder perspectives. Am Health Drug Benefits. 2016;9(9):504-13.

2. Rachakonda TD, Schupp CW, Armstrong AW. Psoriasis prevalence among adults in the United States. J Am Acad Dermatol. 2014;70(3):512-16.

3. Strober BE, van der Walt JM, Armstrong AW, et al. Clinical goals and barriers to effective psoriasis care. Dermatol Ther (Heidelb). 2019;9(1):5-18.

4. Puig L. PASI90 response: the new standard in therapeutic efficacy for psoriasis. J Eur Acad Dermatol Venereol. 2015;29(4):645-48. 
5. Gordon KB, Blauvelt A, Papp KA, et al. Phase 3 trials of ixekizumab in moderateto-severe plaque psoriasis. N Engl J Med. 2016;375(4):345-56.

6. Thaci D, Blauvelt A, Reich K, et al. Secukinumab is superior to ustekinumab in clearing skin of subjects with moderate to severe plaque psoriasis: CLEAR, a randomized controlled trial. J Am Acad Dermatol. 2015;73(3):400-09.

7. Papp K, Thaci D, Reich K, et al. Tildrakizumab (MK-3222), an antiinterleukin-23p19 monoclonal antibody, improves psoriasis in a phase IIb randomized placebo-controlled trial. $\mathrm{Br} \mathrm{J}$ Dermatol. 2015;173(4):930-39.

8. Papp KA, Blauvelt A, Bukhalo M, et al. Risankizumab versus ustekinumab for moderate-to-severe plaque psoriasis. N Engl J Med. 2017;376(16):1551-60.

9. Papp KA, Reich K, Paul C, et al. A prospective phase III, randomized, double-blind, placebo-controlled study of brodalumab in patients with moderateto-severe plaque psoriasis. Br J Dermatol. 2016;175(2):273-86.

10. Blauvelt A, Papp KA, Griffiths CE, et al. Efficacy and safety of guselkumab, an anti-interleukin-23 monoclonal antibody, compared with adalimumab for the continuous treatment of patients with moderate to severe psoriasis: results from the phase III, double-blinded, placebo- and active comparatorcontrolled VOYAGE 1 trial. J Am Acad Dermatol. 2017;76(3):405-17.

11. Reich K, Nestle FO, Papp K, et al. Infliximab induction and maintenance therapy for moderate-to-severe psoriasis: a phase III, multicentre, double-blind trial. Lancet. 2005;366(9494):1367-74.

12. Kouwenhoven TA, van der Ploeg JAM, van de Kerkhof PCM. Treatment goals in psoriasis from a patient perspective: a qualitative study. J Dermatolog Treat. 2020;31(1):13-17.

13. Gorelick J, Shrom D, Sikand K, et al. Understanding treatment preferences in patients with moderate to severe plaque psoriasis in the USA: results from a crosssectional patient survey. Dermatol Ther (Heidelb). 2019;9(4):785-97.
14. Uhlenhake EE, Kurkowski D, Feldman SR. Conversations on psoriasiswhat patients want and what physicians can provide: a qualitative look at patient and physician expectations. J Dermatolog Treat. 2010;21(1):6-12.

15. Armstrong AW, Feldman SR, Korman NJ, et al. Assessing the overall benefit of a medication: cumulative benefit of secukinumab over time in patients with moderate-to-severe plaque psoriasis. J Dermatolog Treat. 2017;28(3):200-05.

16. Brezinski EA, Dhillon JS, Armstrong AW. Economic burden of psoriasis in the United States: a systematic review. JAMA Dermatol. 2015;151(6):651-58.

17. Mattingly TJ 2nd, Levy JF, Slejko JF, Onwudiwe NC, Perfetto EM. Estimating drug costs: how do manufacturer net prices compare with other common us price references? Pharmacoeconomics. 2018;36(9):1093-99.

18. Wu JJ, Feldman SR, Rastogi S, Menges B, Lingohr-Smith M, Lin J. Comparison of the cost-effectiveness of biologic drugs used for moderate-to-severe psoriasis treatment in the United States. J Dermatolog Treat. 2018;29(8):769-74.

19. Al Sawah S, Foster SA, Burge R, et al. Cost per additional responder for ixekizumab and other FDA-approved biologics in moderate-to-severe plaque psoriasis. J Med Econ. 2017;20(12):1224-30.

20. Ravasio R Antonelli S, Maiorino A, Costanzo A, Losi S. Cost per responder for ixekizumab and other biologic drugs approved for the treatment of moderateto-severe plaque psoriasis in Italy. Glob Reg Health Technol Assess. 2019:1-9. doi: $10.1177 / 2284240318822289$

21. Institute for Clinical and Economic Review. Targeted immunomodulators for the treatment of moderate-tosevere plaque psoriasis: effectiveness and value. Final evidence report. August 3, 2018. Accessed December 9, 2020. http://icerorg.wpengine.com/ wp-content/uploads/2020/10/ ICER_Psoriasis_Update_Final_ Evidence_Report_10042018.pdf
22. Warren RB, Gooderham M, Burge R, et al. Comparison of cumulative clinical benefits of biologics for the treatment of psoriasis over 16 weeks: results from a network meta-analysis. J Am Acad Dermatol. 2020;82(5):1138-49.

23. Lebwohl M, Blauvelt A, Paul C, et al. Certolizumab pegol for the treatment of chronic plaque psoriasis: Results through 48 weeks of a phase 3 , multicenter, randomized, double-blind, etanercept- and placebo-controlled study (CIMPACT). J Am Acad Dermatol. 2018;79(2):266-76.e5.

24. Gottlieb AB, Blauvelt A, Thaci D, et al. Certolizumab pegol for the treatment of chronic plaque psoriasis: Results through 48 weeks from 2 phase 3, multicenter, randomized, double-blinded, placebo-controlled studies (CIMPASI-1 and CIMPASI-2). J Am Acad Dermatol. 2018;79(2):302-14.e6.

25. Gordon KB, Strober B, Lebwohl M, et al. Efficacy and safety of risankizumab in moderate-to-severe plaque psoriasis (UltIMMa-1 and UltIMMa-2): results from two double-blind, randomised, placebo-controlled and ustekinumabcontrolled phase 3 trials. Lancet. 2018;392(10148):650-61.

26. NICE Decision Support Unit. Evidence synthesis TSD series. Accessed December 9, 2020. http://nicedsu.org. uk/technical-support-documents/ evidence-synthesis-tsd-series /

27. Dias S, Welton NJ, Sutton AJ, et al. NICE DSU technical support document 2: a generalised linear modelling framework for pairwise and network meta-analysis of randomised controlled trials. April 2014. Accessed December 9, 2020. https://www.ncbi.nlm.nih.gov/books/ NBK310366/

28. Lu G, Ades AE. Combination of direct and indirect evidence in mixed treatment comparisons. Stat Med. 2004;23(20):3105-24.

29. Yu-Sung Su, Masanao Y. R2jags: using R to run 'JAGS'. R package version 0.6-1. 2020. Accessed December 22, 2020. https://CRAN.R-project.org/ package $=$ R2jags 
30. R Core Team. R: a language and environment for statistical computing. 2019. R Foundation for Statistical Computing, Vienna, Austria. Accessed December 22, 2020. https://www.R-project.org/

31. Ades A, Caldwell DM, Reken S, Welton NJ, Sutton AJ, Dias S. NICE DSU technical support document 7: evidence synthesis of treatment efficacy in decision making: a reviewer's checklist. January 2012. Accessed December 9, 2020. http://nicedsu.org.uk/wp-content/ uploads/2016/03/TSD7-reviewerchecklist.final .08.05.12.pdf

32. Brooks SP, Gelman A. General methods for monitoring convergence of iterative simulations. J Comput Graph Stat. 1998;7(4):434-55.

33. Taltz [prescribing information]. Eli Lilly. May 2020. Accessed December 9, 2020. https://pi.lilly.com/us/taltz-uspi.pdf

34. Skyrizi [prescribing information]. AbbVie. April 2019. Accessed December 9, 2020. https://www.accessdata.fda.gov/ drugsatfda docs/label/2019/761105s 000lbl.pdf

35. Humira [prescribing information]. AbbVie. March 2020. Accessed December 9, 2020. https://www.rxabbvie.com/pdf/ humira.pdf

36. Tremfya [prescribing information]. Janssen Biotech. July 2017. Accessed December 9, 2020. https://www. accessdata.fda.gov/drugsatfda docs/ label/2017/761061s000lbl.pdf
37. Cosentyx [prescribing information]. Novartis. January 2018. Accessed December 9, 2020. https://www. accessdata.fda.gov/drugsatfda_docs/ label/2018/125504s013lbl.pdf

38. Cimzia [prescribing information]. UCB. January 2017. Accessed December 9, 2020. https://www.accessdata.fda.gov/drugsatfda docs/label/2017/125160s270lbl.pdf

39. Stelara [prescribing information]. Janssen Biotech. September 2016. Accessed December 9, 2020. https:// www.accessdata.fda.gov/drugsatfda_ docs/label/2016/761044lbl.pdf

40. IBM Watson. IBM Micromedex RED BOOK. Database. Accessed December 9, 2020. https://www.ibm.com/watson/health/provider-client-training/ micromedex-red-book/

41. Reich K, Pinter A, Lacour JP, et al. Comparison of ixekizumab with ustekinumab in moderate-to-severe psoriasis: 24-week results from IXORA-S, a phase III study. Br J Dermatol. 2017;177(4):1014-23. doi: 10.1111/bjd.15666.

42. Blauvelt A, Lomaga M, Burge R, et al. Greater cumulative benefits from ixekizumab versus ustekinumab treatment over 52 weeks for patients with moderateto-severe psoriasis in a randomized, double-blinded phase $3 \mathrm{~b}$ clinical trial. J Dermatolog Treat. 2020;31(2):141-46.

43. Jabbar-Lopez ZK, Yiu ZZN, Ward V, et al. Quantitative evaluation of biologic therapy options for psoriasis: a systematic review and network meta-analysis. J Invest Dermatol. 2017;137(8):1646-54.
44. Xu G, Xia M, Jiang C, et al. Comparative efficacy and safety of thirteen biologic therapies for patients with moderate or severe psoriasis: a network meta-analysis. J Pharmacol Sci. 2019;139(4):289-303.

45. Armstrong A, Jarvis S, Boehncke WH, et al. Patient perceptions of clear/almost clear skin in moderate-to-severe plaque psoriasis: results of the Clear About Psoriasis worldwide survey. J Eur Acad Dermatol Venereol. 2018;32(12):2200-07.

46. Feldman SR, Foster SA, Zhu B, Burge R, Al Sawah S, Goldblum OM. Cost per additional responder associated with ixekizumab and etanercept in the treatment of moderate-to-severe psoriasis. J Drugs Dermatol. 2017;16(12):1246-52.

47. Teeple A, Muser E. Cost per response for guselkumab versus adalimumab in the United States using data from a head-tohead trial in moderate-to-severe plaque psoriasis. J Med Econ. 2019;22(12):1268-73.

48. Malatestinic W, Nordstrom B, Wu JJ, et al. Characteristics and medication use of psoriasis patients who may or may not qualify for randomized controlled trials. J Manag Care Spec Pharm. 2017;23(3):37081. doi: $10.18553 /$ jmcp.2017.16367

49. Phillippo DM, Dias S, Ades AE, et al. Multilevel network meta-regression for population-adjusted treatment comparisons. J R Statist Soc A. 2020;183(3):1189-210. 